\title{
VERBOWEB: UMA PROPOSTA DE CLASSIFICAÇÃO VERBAL ${ }^{1}$
}

\section{VERBOWEB:A PROPOSAL FOR VERB CLASSIFICATION}

\author{
Márcia Cançado \\ Universidade Federal de Minas Gerais \\ Belo Horizonte, Minas Gerais Brasil \\ Luana Amaral \\ Universidade Federal de Minas Gerais \\ Belo Horizonte, Minas Gerais Brasil \\ Letícia Lucinda Meirelles \\ Universidade Federal de Minas Gerais \\ Belo Horizonte, Minas Gerais Brasil
}

\begin{abstract}
RESUMO: O VerboWeb é um banco de dados lexicais do português brasileiro, em desenvolvimento, que conta atualmente com mais de 1360 verbos classificados em classes e em subclasses, de acordo com suas propriedades sintáticosemânticas. Neste artigo, apresentamos uma proposta de classificação verbal por propriedades léxico-gramaticais. Mostramos como se deu a classificação verbal utilizada no VerboWeb e os possíveis níveis de classificação dos verbos. Propomos que são necessárias para a descrição e análise do léxico verbal do português brasileiro dois níveis de classificação: classes e subclasses. Explicitamos a que nível de granularidade estão associadas as classes e subclasses do banco.
\end{abstract}

PALAVRAS-CHAVE: Interface Sintaxe-Semântica Lexical; Classificação verbal; Níveis de classificação

\begin{abstract}
VerboWeb is a lexical database of Brazilian Portuguese, in development, which currently has more than 1360 verbs classified in classes and subclasses, according to their syntactic-semantic properties. In this paper, we present a proposal of verb classification by lexico-grammatical properties. We show how the verb classification used in VerboWeb was made and the possible levels of analysis. We propose that two levels of classification are necessary for the description and analysis of the verbal lexicon of Brazilian Portuguese: classes and subclasses. We explain to what level
\end{abstract}

1 A autora Márcia Cançado agradece ao apoio financeiro do CNPq (bolsa de produtividade) e da FAPEMIG (auxílio PPM). A autora Letícia Lucinda Meirelles agradece à FAPEMIG pela bolsa de doutorado. 
of granularity the classes and subclass of the database are associated.

KEYWORDS: Syntax-Lexical Semantics Interface; Verb classification; Levels of classification 


\section{INTRODUÇÃO}

Os itens lexicais de uma língua podem ser classificados de acordo com diferentes critérios. As classes de palavras da Gramática Tradicional (substantivos, verbos, advérbios, etc.) são um bom exemplo de como o léxico pode ser organizado e categorizado. Como observam De Clerck, Colleman e Willems (2013), os verbos, de maneira especial, têm recebido muita atenção na literatura a esse respeito, e são, eles também, internamente classificados, de diferentes formas. Por exemplo, ainda na Gramática Tradicional, eles são divididos em grupos de acordo com os complementos que aceitam ou não. Assim, os verbos são categorizados em quatro grupos: intransitivos (sem complemento: nascer), transitivos diretos (com um objeto direto: comer algo), transitivos indiretos (com um objeto indireto: gostar de algo) e transitivos diretos e indiretos (com um objeto direto e um indireto: colocar algo em algum lugar).

Além disso, há também classificações feitas com base em outros critérios, como, por exemplo, a classificação aspectual de Vendler (1967), que leva em conta a maneira como a situação descrita pelo verbo se desenrola no decorrer do tempo. Nessa classificação, os verbos podem ser estados (as situações não mudam ao longo do tempo: ter uma casa), atividades (as situações mudam ao longo do tempo, mas não atingem um ponto final específico: correr), accomplishments (as situações mudam ao longo do tempo e atingem um ponto final específico: construir uma casa) ou achievements (situações pontuais que ocorrem quase instantaneamente: estourar $)^{2}$.

Existe, ainda, outro tipo de classificação verbal que utiliza um critério chamado por De Clerck, Colleman e Willems (2013) de "propriedades léxicogramaticais". Segundo os autores, esse tipo de classificação é "motivado pela descoberta de que verbos com significados semelhantes apresentam uma tendência a possuírem o mesmo comportamento sintático" (DE CLERCK, COLLEMAN; WILlEMS, 2013, p. 669) ${ }^{3}$. Também nesse sentido, Cançado, Godoy e Amaral $(2013$, 2017) propõem que classes de verbos podem ser definidas a partir de propriedades semânticas que tenham impacto na sintaxe. Esse tipo de classificação é baseado na hipótese da determinação semântica sobre a sintaxe (FILLMORE, 1970; PINKER, 1989; JACKENDOFF, 1990; LEVIN, 1993; PESETSKY, 1995; GRIMSHAW, 2005; LEVIN; RAPPAPORT HOVAV, 2005; CANÇADO; GODOY; AMARAL, 2013, 2017; CANÇADO; AMARAL, 2016), assumida por muitos semanticistas lexicais e que diz que partes do significado de um verbo são relevantes para a realização sintática de seus argumentos. Baseadas, então, nesse último critério, é que propomos a classificação verbal aqui apresentada.

\footnotetext{
${ }^{2}$ Os termos accomplishment e achievement são convencionalmente usados em inglês na literatura em português brasileiro.

${ }^{3}$ Traduzido por nós do original: "fuelled by the discovery that verbs with a similar meaning show a tendency toward displaying the same syntactic behavior".
} 
Exemplos de classes definidas a partir desse critério são a classe dos verbos de mudança de estado (quebrar, rasgar) (FILLMORE, 1970; PARSONS, 1990; LEVIN, 1993; CANÇADO; AMARAL, 2010; dentre muitos outros) e a classe de verbos instrumentais (serrar, chicotear) (KIPARSKY, 1982; LEVIN, 1993; HARLEY, 2005; MEIRELLES; CANÇADO, 2015). O primeiro grupo, o da classe de verbos de mudança de estado, é composto por verbos que descrevem uma mudança de estado do argumento em posição de objeto direto, causada (com intenção ou não) pelo argumento na posição de sujeito:

(1) O vândalo quebrou a estátua do prefeito.

(2) O dançarino rasgou as cortinas do teatro.

Os verbos que apresentam essa propriedade semântica, uniformemente, aceitam certas propriedades sintáticas. Por exemplo, são todos transitivos diretos e aceitam a chamada "alternância causativo-incoativa" (LAKOFF, 1970; FILLMORE, 1970; LEVIN; RAPPAPORT-HOVAV, 1995; CANÇADO; AMARAL, 2010), uma alternância de argumentos em que o item que ocupa a posição de objeto direto passa a ocupar a posição de sujeito:

(3) A estátua do prefeito (se) quebrou.

(4) As cortinas do teatro (se) rasgaram.

Já, o segundo grupo, o dos verbos instrumentais, compartilha a semântica de um evento necessariamente mediado pelo instrumento que dá nome ao verbo: serra para serrar e chicote para chicotear.

(5) O artesão serrou a madeira.

(6) O fazendeiro chicoteava o gado.

Esses verbos, uniformemente, são transitivos diretos, não aceitam a alternância causativo-incoativa (como mostram os exemplos em (7) e (8) abaixo) e aceitam a inserção de um sintagma preposicionado cognato, que especifica o tipo do instrumento usado no evento (como mostram os exemplos em (9) e (10)):

(7) *A madeira (se) serrou.

(8) *O gado (se) chicoteava.

(9) O artesão serrou a madeira com uma serra velha.

(10) O fazendeiro chicoteava o gado com um chicote de aço.

Entretanto, a hipótese da determinação semântica sobre a sintaxe não se aplica de uma forma indiscriminada a qualquer propriedade semântica, ou seja, não são todas as propriedades semântico-lexicais dos verbos que possuem 
impacto na sintaxe. Portanto, não é qualquer agrupamento semântico de verbos que constitui uma classe verbal, nessa perspectiva.

Levin e Rappaport Hovav (1992), por exemplo, mostram que os verbos que acarretam movimento para um de seus argumentos não se comportam sintaticamente da mesma maneira. Verbos como correr, balançar e jogar podem ser igualmente considerados verbos de movimento, pois todos acarretam que um de seus argumentos se move de alguma forma:

\section{(11) O maratonista correu.}

(12) O menino balançou a cortina.

(13) O atacante arremessou a bola para o gol.

Porém, o verbo correr é intransitivo; já o verbo balançar é transitivo e o verbo arremessar é bitransitivo. O simples fato de os verbos não apresentarem a mesma transitividade já é uma boa evidência de que a propriedade semântica 'movimento' não tem impacto na sintaxe $\mathrm{e}$, portanto, não será válida para a classificação verbal baseada no critério de propriedades léxico-gramaticais. É trabalho do semanticista lexical, portanto, encontrar quais propriedades semânticas determinam o comportamento sintático dos verbos e, a partir delas, determinar a classificação desses itens da língua.

Neste artigo, adotando o tipo de classificação verbal baseado no critério das propriedades léxico-gramaticais, propomos uma classificação dos verbos do português brasileiro (PB) em classes e subclasses, estabelecendo níveis de análise a partir das propostas teóricas sobre granularidade de Levin (2010) e Cançado e Gonçalves (2016). Nossa proposta explicita a análise do léxico verbal que vem sendo desenvolvida no "Banco de Dados Lexicais VerboWeb: classificação sintático-semântica dos verbos do português brasileiro" (CANÇADO; AMARAL; MEIRELLES, 2017) ${ }^{4}$. O projeto conta com um número abrangente de verbos e suas classificações de acordo com propriedades sintáticas e semânticas (atualmente estão disponíveis no banco mais de 1360 verbos).

$\mathrm{O}$ artigo está dividido da seguinte forma: na próxima seção, apresentamos o aporte teórico para a classificação verbal que propomos: a noção de níveis de análise das classes verbais proposta em Cançado e Gonçalves (2016); na terceira seção, apresentamos nossa proposta de classificação baseada em classes e subclasses; na quarta seção, mostramos o procedimento de classificação verbal adotado no VerboWeb; por fim, a última seção traz a síntese de nosso trabalho.

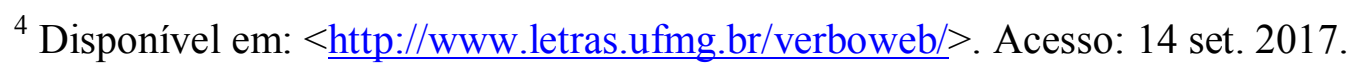




\section{NÍVEIS DE CLASSIFICAÇÃO: A PROPOSTA DE GRANULARIDADE DE CANÇADO E GONÇALVES (2016)}

A partir do trabalho de Levin (2010), dentro da perspectiva de classificação verbal por propriedades léxico-gramaticais, Cançado e Gonçalves (2016) propõem que a classificação pode variar de acordo com o nível de análise (grain size) adotado, com a chamada "granularidade" da análise (DE CLERCK; COLLEMAN; WILLEMS, 2013). Para as autoras, são três os níveis de classificação: coarse-grained, que corresponde a um nível mais amplo; mediumgrained, que corresponde ao nível intermediário; e fine-grained, que diz respeito a um agrupamento feito a partir de propriedades mais finas/restritas dos verbos.

O que comumente se denomina de "classe verbal" na literatura em Semântica Lexical refere-se à classificação intermediária, medium-grained. Um exemplo de classe no nível medium-grained (classe verbal canônica), segundo Cançado e Gonçalves (2016), é a dos verbos de mudança de estado do PB, já apresentada anteriormente. Essa classe é composta por verbos que compartilham propriedades semânticas e que estão associados a uma série de propriedades sintáticas em comum, como a participação na alternância causativo-incoativa, mostrada nos exemplos (3) e (4).

Assume-se que os verbos de uma classe medium-grained apresentam a mesma estrutura argumental. Aqui, a estrutura argumental é entendida como a representação das propriedades semântico-lexicais dos verbos que são importantes para a organização sintática. No caso dos verbos de mudança de estado, pode-se assumir, de acordo com Cançado, Godoy e Amaral (2013, 2017), que os verbos pertencentes a essa classe possuem a seguinte estrutura argumental, em que os parênteses indicam a possibilidade de intencionalidade na ação do argumento na posição de sujeito:

$$
\text { v: }\{\text { Causa (Agente), Paciente }\}^{5}
$$

Uma análise no nível coarse-grained, por sua vez, leva em conta propriedades mais amplas dos verbos que não são restritas a uma única classe medium-grained. Assim, classes coarse-grained são agrupadas por propriedades comuns a diferentes estruturas argumentais. Como exemplo, Cançado e Gonçalves (2016) apontam os verbos agentivos do PB.

Lavar: \{Agente, Paciente\}

a. A lavadeira lavou os lençóis de algodão.

b. Os lençóis de algodão foram lavados.

\footnotetext{
5 Essa representação também pode se dar por outros tipos de linguagem, como por exemplo, em uma estrutura de decomposição de predicados. Deixaremos aqui a estrutura em termos de papéis temáticos por ser uma representação mais utilizada em várias abordagens teóricas.
} 
(16) Lançar: \{Agente, Tema, Locativo\}

a. $\mathrm{O}$ atleta lançou o dardo para cima.

b. O dardo foi lançado.

(17) Cravar: $\{$ Agente, Paciente, Locativo\}

a. O biólogo cravou a placa no pé da árvore.

b. A placa foi cravada no pé da árvore.

De acordo com Cançado, Amaral e Meirelles (2017), os verbos de (15) a (17) pertencem cada um a uma classe do tipo medium-grained distinta. Porém, todos apresentam o papel temático de Agente na posição de sujeito e aceitam a forma passiva (JACKENDOFF, 1972). Portanto, a passivização é uma propriedade sintática que motiva uma classificação coarse-grained, pois agrupa em uma classe ampla todos os verbos transitivos agentivos do PB.

Por fim, uma análise em nível fine-grained diz respeito ao agrupamento de verbos por propriedades específicas de seu sentido, ou seja, por propriedades bem finas que não estão presentes em sua estrutura argumental, mas ainda assim estão ligadas diretamente a um (ou mais) tipo de propriedade sintática. Cançado e Gonçalves (2016) apontam como exemplo de uma classificação fine-grained os chamados verbos recíprocos (FONSECA, 1984; DIXON, 1992; SILONI, 2007; GODOY, 2009, 2010) do PB. Esses verbos apresentam como propriedades semânticas comuns o fato de terem um argumento plural e denotarem uma relação de reciprocidade entre os referentes desse argumento e como propriedade sintática comum o fato de poderem se alternar entre uma forma simples e uma descontínua (GODOY, 2009, 2010), como mostramos a seguir:

(18) a. A briga pela herança do pai separou os irmãos. $\rightarrow$ forma simples

b. A briga pela herança do pai separou um irmão do outro. $\rightarrow$ forma descontínua

(19) a. As meias da menina combinam. $\rightarrow$ forma simples

b. Uma meia combina com a outra. $\rightarrow$ forma descontínua

Como Cançado, Godoy e Amaral (2013, 2017) apontam, os verbos recíprocos do $\mathrm{PB}$ não formam uma classe medium-grained. Alguns, como separar, encontram-se na classe dos verbos de mudança de estado transitivos, apresentando a estrutura argumental v: \{Causa (Agente), Paciente\}, e outros, como combinar, são basicamente intransitivos, não fazendo parte dessa classe, e apresentando a estrutura argumental v: \{Objeto Estativo\}, já que são verbos que descrevem situações estáticas (MOREIRA, 2000; BECHIR, 2017). Não há, assim, nenhum elemento da estrutura argumental desses verbos que seja capaz de os agrupar. Também, a reciprocidade não está associada a nenhum papel temático específico, impedindo, portanto, que essa seja uma classificação do nível coarsegrained. Dessa forma, a reciprocidade é uma propriedade dos verbos que motiva uma análise no nível fine-grained.

É importante ressaltar, como já apontamos, que a proposta de Cançado e Gonçalves (2016) se baseia em um tipo de classificação que se vale de propriedades léxico-gramaticais como critério: independentemente da 
granularidade da classe, de quão ampla ou específica ela é, a classificação é sempre feita a partir de propriedades semânticas que possuem impacto na sintaxe.

\section{UMA PROPOSTA DE CLASSIFICAÇÃO PARA OS VERBOS DO PB: CLASSES E SUBCLASSES}

De Clerck, Colleman e Willems (2013) discutem em seu trabalho a importância de se analisar a granularidade mais adequada para os propósitos da pesquisa a ser realizada. Qual grain-size é o mais útil ou mais adequado a ser adotado na classificação verbal de uma língua? Acreditamos que todos os tipos de análise são possíveis e úteis, porém, para os propósitos de classificação do projeto VerboWeb, propomos que são essenciais dois níveis de classificação: as classes canônicas, em um nível de classificação medium-grained, e suas subclasses, em um nível de análise fine-grained.

As classes (em nível medium-grained, daqui para frente chamadas somente de "classes") são aqui definidas, seguindo a proposta de Cançado e Gonçalves (2016), como grupos de verbos que compartilham toda a sua estrutura argumental. Essas classes são as centrais na língua, pois esses agrupamentos de verbos são os que compartilham o maior número de propriedades sintáticas. Retomando o exemplo dado para esse tipo de classe, os verbos de mudança de estado, além de compartilharem a propriedade de possibilitar a alternância causativo-incoativa, eles também: (I) licenciam a inserção do argumento causa em adjunção na forma intransitiva, (II) licenciam a inserção de um instrumento e (III) licenciam passiva com um agente (CANÇADO; GODOY; AMARAL, 2013, 2017):

(20) a. A estátua do prefeito (se) quebrou (com a queda).

b. O vândalo quebrou a estátua do prefeito com uma marreta.

c. A estátua do prefeito foi quebrada pelo vândalo.

a. As cortinas do teatro (se) rasgaram (com o vendaval).

b. O dançarino rasgou as cortinas do teatro com uma tesoura.

c. As cortinas do teatro foram rasgadas pelo dançarino.

As classes em nível coarse-grained, diferentemente, compartilham poucas (ou apenas uma) propriedade. Além disso, por estarem associadas à estrutura argumental dos verbos, essas propriedades também são das classes. Portanto, a classificação nesse nível mais amplo se torna desnecessária e não muito útil do ponto de vista de um projeto como o VerboWeb, que tem como objetivo fazer uma ampla análise do léxico verbal do PB. O caso da passivização, por exemplo, ilustra bem essa questão. A passivização, que motiva uma classificação em nível coarse-grained, por ser uma propriedade relacionada à estrutura argumental dos verbos, aparecerá como propriedade de todas as classes que tenham um agente representado em sua estrutura argumental.

Outro exemplo de classe coarse-grained é a que reúne verbos do tipo acorrentar a verbos do tipo chicotear. 
(22) O engenheiro de segurança acorrentou os portões da casa com correntes de ferro.

(23) O patrão chicoteou o escravo com um chicote de espinhos.

(CANÇADO; AMARAL; MEIRELLES, 2017)

Como podemos observar nos exemplos (22) e (23), ambos os verbos aceitam a inserção de um sintagma preposicionado cognato que denota instrumento. Contudo, eles pertencem a classes medium-grained diferentes: o verbo chicotear pertence à classe dos verbos instrumentais, que será descrita na seção seguinte, enquanto o verbo acorrentar pertence à classe dos verbos de mudança de posse. Além de apresentarem aspectos lexicais distintos (os verbos do tipo chicotear denotam atividades, enquanto os do tipo acorrentar denotam accomplishments), o verbo acorrentar lexicaliza a noção de mudança de posse (o portão é provido com correntes de ferro), enquanto o verbo chicotear lexicaliza a afetação de uma entidade por meio de um instrumento, sem que esse passe a ficar sob posse da entidade afetada.

Uma classificação em nível fine-grained é também interessante para os propósitos de classificação do VerboWeb. Ao contrário das propriedades amplas das classes coarse-grained, as propriedades das classes fine-grained não estão associadas às estruturas argumentais dos verbos. Portanto, essas propriedades não aparecerão nas classes. Assim, vários outros aspectos relevantes da língua são captados se incluirmos esse nível em nossa análise.

Propomos, portanto, que, dentro de cada classe, é possível que existam verbos que se agrupem por propriedades semânticas e sintáticas mais específicas. Esses agrupamentos são classes em um nível fine-grained e são chamados de "subclasses". Cada subclasse de verbos pertence a uma única classe e os verbos que pertencem a cada subclasse possuem todas as propriedades semânticas e sintáticas da classe e mais propriedades específicas que os agrupam na subclasse. Retomando nosso exemplo, os verbos recíprocos se subdividem em duas subclasses: uma que faz parte da classe dos verbos de mudança de estado (tipo separar) e outra que faz parte dos verbos estativos (tipo combinar). Cada um desses verbos possui, respectivamente, todas as propriedades dos verbos de mudança de estado e dos verbos estativos, mais as propriedades dos verbos recíprocos.

A divisão dos verbos de uma língua por classes e subclasses não é novidade na Semântica Lexical. O amplo trabalho de classificação dos verbos do inglês feito por Levin (1993) já traz essa ideia. No trabalho da autora, encontramos diferentes classes divididas em subclasses, de acordo com especificidades de cada agrupamento. Apesar de também nos inspirarmos no trabalho de Levin (1993) e de assumirmos grande parte de seus pressupostos teóricos, a nossa classificação se distingue da adotada pela autora em um ponto importante: em nossa proposta, todos os verbos de uma subclasse compartilham as propriedades gerais da classe e possuem propriedades adicionais da subclasse. Para Levin (1993), subclasses dentro de uma classe podem ser distintas e sem relação com as propriedades gerais da classe, entretanto apresentam algum tipo de sentido em comum. 
Um bom exemplo dessa distinção está na classificação dos verbos de remoção (LEVIN; RAPPAPORT HOVAV, 1991; LEVIN, 1993; ALEXIADOU; ANAGNOSTOPOULOU, 2013; ALMEIDA, 2015; OLIVEIRA, 2016). Para Levin (1993), os verbos de remoção constituem-se como uma classe, na qual estão inseridas, entre outras, as subclasses remove verbs (remover) e wipe verbs (esfregar). Não existem propriedades sintáticas comuns aos verbos de remoção, de modo que a autora agrupa esses verbos apenas pelo fato de apresentarem a propriedade semântica 'remoção'. Já as subclasses são definidas por propriedades sintáticas, mais especificamente alternâncias. Os verbos do tipo wipe verbs, por exemplo, segundo a autora, aceitam a alternância chamada de locativa (o que ocorre também em português; OLIVEIRA, 2016). Esses verbos podem ter uma estrutura em que há um sentido de remoção, como em (a), e outro em que há apenas uma ação sobre um paciente, como em (b):

a. A faxineira esfregou a sujeira do chão.

b. A faxineira esfregou o chão. (CANÇADO; AMARAL; MEIRELLES, 2017)

Já os verbos do tipo remove verbs não aceitam essa alternância, podendo ocorrer somente em uma estrutura como em (a):

(25) a. A faxineira removeu a sujeira do tapete.

b. *A faxineira removeu o tapete. ${ }^{6}$

Em nossa análise, existem dois tipos de verbos de remoção. O primeiro, que comporta verbos como esfregar, tosar e varrer, é uma subclasse que faz parte da classe dos verbos de atividade com afetação (CANÇADO; AMARAL; MEIRELLES, 2017). Todos esses verbos, incluindo os que não são da subclasse de remoção, acarretam que a ação foi realizada por um instrumento (HASPELMATH, 1993), licenciam um instrumento na posição de sujeito, licenciam a forma passiva com um agente, licenciam apagamento do objeto (com implicatura de um objeto prototípico), licenciam uma alternância sintática com leitura de genericidade chamada medial ou média (KEYSER; ROEPER, 1984; LEVIN; RAPPAPORT-HOVAV, 1994) e não licenciam alternância causativoincoativa. Exemplos de verbos dessa classe que não denotam o sentido de remoção são os verbos moer, afiar e picotar. A seguir exemplificamos as propriedades da classe, na ordem em que foram listadas, para os verbos moer e esfregar.

(26) a. O açougueiro moeu a carne com o processador.

b. O processador do açougue moeu a carne muito bem.

c. A carne foi moída pelo açougueiro.

d. Processador moe melhor que moedor manual.

e. Patinho moe fácil.

f. *A carne (se) moeu.

(27) a. A faxineira esfregou o chão com um esfregador elétrico.

\footnotetext{
${ }^{6}$ Agramatical na mesma interpretação de (a).
} 
b. O esfregador elétrico esfregou o chão rapidamente.

c. O chão foi esfregado bem rapidamente pela faxineira.

d. A faxineira lá de casa esfrega, lava e encera muito bem.

e. Chão de porcelanato esfrega rápido.

f. *O chão (se) esfregou.

(CANÇADO; AMARAL; MEIRELLES, 2017)

Verbos como moer não aceitam a alternância locativa. Verbos de remoção (esfregar) que são da classe de moer, entretanto, além de exibirem todas essas propriedades, ainda podem participar da alternância locativa (como mostra o exemplo (24)).

O segundo tipo de verbos de remoção não forma uma classe, nem uma subclasse, em nossa proposta, como mostra Almeida (2015). Esses verbos, do tipo de remover, se comportam como os verbos chamados de verbos de mudança de estado locativo (LEVIN, 1993; CANÇADO; GODOY; AMARAL, 2013, 2017). Os verbos de mudança de estado locativo são bitransitivos, licenciam passiva com locativo e não licenciam a alternância causativo-incoativa. Um bom exemplo é o verbo guardar:

(28) a. O motoqueiro guardou a jaqueta (no armário).

b. A jaqueta foi guardada no armário.

c. *A jaqueta se guardou no armário.

(CANÇADO; AMARAL; MEIRELLES, 2017)

Remover e outros verbos similares que trazem a ideia de remoção possuem todas essas propriedades, porém não possuem mais outras propriedades específicas decorrentes do sentido de remoção.

(29) a. A manicure removeu o esmalte (da unha da Joana).

b. O esmalte foi removido da unha da Joana.

c. *O esmalte se removeu da unha da Joana.

(CANÇADO; AMARAL; MEIRELLES, 2017)

Assim, em nossa análise, essa propriedade, nesse caso, não motiva a classificação em nenhum nível.

Em conclusão, propomos que a granularidade adequada para análise no caso do projeto VerboWeb é a representada nas classes e subclasses, cada uma correspondente a um nível de análise. As classes correspondem ao nível mediumgrained (mesma estrutura argumental), enquanto as subclasses correspondem ao fine-grained (mesma propriedade semântica específica). Cada subclasse é associada a uma classe, de modo que os verbos pertencentes a uma subclasse apresentam as mesmas propriedades sintáticas da classe à qual a subclasse está relacionada e mais alguma(s) propriedade(s) adicional(is) decorrente(s) de seu sentido específico. A seguir, explicitamos o funcionamento dessa classificação através de uma série de exemplos de classes e suas respectivas subclasses no VerboWeb. 


\section{CLASSIFICAÇÃO NO VERBOWEB}

Para mostrar o processo de classificação adotado no VerboWeb, começamos pelos chamados "verbos de atividade inergativos". Segundo Amaral e Cançado (2015), esses são verbos intransitivos agentivos que denotam a realização de um evento; Cançado, Amaral e Meirelles (2017) atribuem a eles seguinte estrutura argumental: $v$ : \{Agente\}. As propriedades sintáticas apresentadas por esses verbos são: (I) são basicamente intransitivos, (II) aceitam um objeto cognato que denota um evento, (III) licenciam a inserção de um adjunto equivalente ao objeto cognato e (IV) apresentam um sintagma nominal correlato ao sentido verbal acrescido de uma expressão temporal, o que evidencia o caráter eventivo do objeto cognato. Todas essas propriedades são apresentadas a seguir na ordem em que foram listadas.
a. $\mathrm{O}$ atleta corria sempre.
b. $\mathrm{O}$ atleta correu uma corrida perfeita.
c. $\mathrm{O}$ atleta correu perfeitamente.
d. A corrida do atleta durou duas horas.
a. $\mathrm{O}$ bebezinho sorria demais.
b. O bebezinho sorriu um sorriso doce.
c. O bebezinho sorriu docemente.
d. O sorriso do bebezinho durou pouco.

(CANÇADO; AMARAL; MEIRELLES, 2017 )

É importante ressaltar que o objeto cognato não é um argumento do verbo. Esse elemento tem a função de especificar o evento denotado pelo verbo, sendo composto de um nome cognato com um modificador, de modo que a modificação atribuída ao nome pode ser transferida para o verbo (HORROCKS; STAVROU, 2010; SHER, LEUNG, 2006), como pode ser observado nos exemplos (b) e (c) das sentenças em (30) e (31).

Vejamos agora um tipo de subclasse associada à classe dos verbos de atividade inergativos. Tomemos o verbo fofocar, classificado na literatura como sendo um verbo de expressão (LEHRER, 1988; LEVIN, 1993; PESETSKY, 1995; SARTORI, 2017). Esse verbo, além de apresentar as propriedades da classe dos verbos de atividade inergativos (da letra (a) a (d)), também apresenta outras propriedades adicionais (da letra (e) a (h)).

(32) a. As vizinhas daquele condomínio fofocavam muito.

b. As vizinhas fofocaram fofocas maliciosas.

c. As vizinhas fofocaram maliciosamente.

d. As fofocas das vizinhas duraram a tarde toda.

e. As vizinhas sempre fofocavam as novidades do bairro.

f. A vizinha fofocava as novidades do bairro para a mãe.

g. A vizinha fofocava sempre para a mãe.

$\mathrm{h}$. A vizinha maldosa fofocou que o marido da amiga saía escondido.

(CANÇADO; AMARAL; MEIRELLES, 2017) 
Segundo Cançado, Amaral e Meirelles (2017), é o sentido específico do verbo fofocar (denotar uma expressão), que the permite realizar as propriedades exemplificadas em (32e) a (32h). Portanto, o sentido de 'denotar um tipo de expressão' agrupa os verbos em nível fine-grained, formando o que denominamos de subclasse. As propriedades sintáticas dos verbos que pertencem a essa subclasse são: (I) licenciam a presença de um objeto que denota o conteúdo da expressão (32e); (II) aceitam um objeto direto denotando o conteúdo de expressão acrescido de um sintagma preposicionado alvo (32f); (III) licenciam apenas o sintagma preposicionado alvo do conteúdo da expressão (32g); (IV) e aceitam um objeto sentencial (32h).

Retomando a ideia, por apresentarem essas propriedades sintáticas adicionais, os verbos de expressão formam uma subclasse da classe dos verbos de atividade inergativos. Essa subclasse é chamada de "verbos de expressão" e os verbos que a compõem exibem as mesmas propriedades gerais da classe mediumgrained (exemplos em 30, 31 e em 32 de (a) a (d)) e mais outras propriedades específicas, exemplificadas de (32e) a (32h). A classe dos verbos de atividade inergativos apresenta um total de 119 verbos e a subclasse dos verbos de expressão é composta por 21 verbos.

Vejamos agora o caso do verbo dizer. Ele também é um verbo de expressão e, portanto, deveria apresentar as mesmas propriedades dessa subclasse. Porém, isso não é completamente verdadeiro, como podemos ver nas sentenças a seguir:

(33) a. Os políticos sempre disseram muita mentira.

b. Os políticos disseram mentiras para os seus eleitores.

c. Os políticos disseram que melhorariam o país.

d. *Os políticos disseram para os eleitores.

$\mathrm{O}$ verbo dizer, assim como os do tipo fofocar, licencia a presença de objeto sentencial (33c) e aceita um objeto direto (mentira $(s))$ que denota o conteúdo da expressão e que pode vir acrescido de um alvo (para os seus eleitores). Contudo, diferentemente da subclasse de fofocar, dizer não permite que o alvo seja expresso sem o constituinte que denota o conteúdo da expressão (33d). Além disso, também não apresenta uma forma intransitiva: *os políticos diziam sempre. Isso ocorre porque esse verbo não pertence à classe medium-grained dos verbos de atividade inergativos e, por isso, não faz parte da mesma subclasse que fofocar, gritar e cantar.

Para finalizar, retomemos o exemplo da classe dos verbos instrumentais. Cançado, Amaral e Meirelles (2017) denominam essa classe de "verbos de atividade com afetação (instrumentais)", pois os verbos denotam a afetação de seu argumento interno por meio do uso de um instrumento, apresentando a seguinte estrutura argumental: \{Agente, Paciente\}. $\mathrm{O}$ instrumento utilizado na ação é que dá nome ao verbo. Como mostramos nos exemplos de (7) a (10),

\footnotetext{
${ }^{7}$ De acordo com Sartori (2017) existem 15 verbos do PB que se comportam como dizer. São verbos como descrever, pronunciar, proferir, etc.
} 
repetidos abaixo, os verbos dessa classe não participam da alternância causativaincoativa e aceitam a inserção de um sintagma preposicionado cognato:

*A madeira (se) serrou.

*O gado (se) chicoteava.

$\mathrm{O}$ artesão serrou a madeira com uma serra velha.

O fazendeiro chicoteava o gado com um chicote de aço.

Contudo, existem alguns verbos que exibem, além dessas propriedades, a possibilidade de apresentar um outro instrumento como adjunto, desde que esse seja uma espécie de intermediário entre o agente e o instrumento que nomeia o verbo.

(38) O bandido baleou a vítima com um revólver calibre 38.

(39) O povo apedrejou a Maria Madalena com estilingues.

(CANÇADO; AMARAL; MEIRELLES, 2017)

Nas sentenças acima, os adjuntos instrumentos com um revólver calibre 38 e com estilingues são intermediadores entre o agente e os instrumentos que nomeiam os verbos: bala e pedra, respectivamente. Assim, os verbos balear e apedrejar denotam eventos que podem ser realizados através de uma cadeia de instrumentos: o primeiro instrumento (revólver/ estilingue) é utilizado pelo agente para lançar o instrumento que denomina o verbo (balalpedra) e que afeta o argumento interno verbal (a vítimal a Maria Madalena). Os verbos instrumentais que apresentam esse sentido adicional são classificados como uma subclasse, chamada de "verbos instrumentais com dois instrumentos", dos verbos de atividade com afetação (instrumentais). Fazem parte da classe 28 verbos e da subclasse, 7 verbos.

Com as classes dos verbos de atividade inergativos e dos verbos de atividade com afetação (instrumentais), exemplificamos como é feita a classificação no VerboWeb por meio na noção de "classe", ou seja, por uma classificação por propriedades léxico-gramaticais em um nível medium-grained. Atualmente, o banco conta com mais de 1360 verbos, divididos em 12 classes. As subclasses de verbos de expressão e de verbos instrumentais com dois instrumentos exemplificam o trabalho de classificação feito em um nível finegrained, que, como mostramos, é também relevante para a descrição e para uma análise teórica do Léxico verbal do PB ou de qualquer outra língua. Todos os verbos se encaixam em alguma classe, porém não são todos os verbos que pertencem a alguma subclasse. Atualmente, o banco conta com a análise de cerca de 380 verbos, divididos em 15 subclasses. Propomos que esse tipo de classificação, em classes e subclasses, e por propriedades léxico-gramaticais, apresenta a forma mais adequada de descrição e análise do Léxico verbal do PB, para os propósitos do VerboWeb. 


\section{CONSIDERAÇÕES FINAIS}

Neste artigo, apresentamos uma proposta de classificação para os verbos do $\mathrm{PB}$, assumindo como critério as propriedades léxico-gramaticais e tomando como aporte teórico a proposta de granularidade de Cançado e Gonçalves (2016). As autoras propõem que são relevantes três níveis de análise na classificação verbal. Um nível coarse-grained, mais amplo, definido por propriedades semânticas que ocorrem em distintas estruturas argumentais dos verbos e que têm algum impacto na sintaxe, um nível medium-grained, definido por todas as propriedades compartilhadas na estrutura argumental dos verbos, e um nível finegrained, definido por propriedades semânticas comuns dos verbos que não se encontram na estrutura argumental, mas que impactam a sintaxe de alguma forma. Em nossa proposta, assumimos dois níveis de análise: classes e subclasses. As classes são aqui definidas como grupos de verbos que compartilham toda a sua estrutura argumental, em um nível medium-grained. Essas classes são as centrais na língua, pois são os agrupamentos de verbos que compartilham o maior número de propriedades sintáticas. Não assumimos a classificação em nível coarsegrained, já que essas propriedades, por estarem associadas à estrutura argumental dos verbos, também são das classes. Propomos, ainda, que, dentro de cada classe, é possível que existam verbos que se agrupem por propriedades semânticas e sintáticas mais específicas. Esses agrupamentos são classes em um nível finegrained e são chamados de subclasses. Cada subclasse de verbos pertence a uma única classe e os verbos que pertencem a cada subclasse possuem todas as propriedades semânticas e sintáticas da classe e mais propriedades específicas que os agrupam na subclasse.

Utilizamos o banco de dados lexicais VerboWeb para ilustrar os possíveis níveis de classificação verbal que propomos. Mostramos duas classes presentes no banco e suas propriedades semânticas e sintáticas: os verbos de atividade inergativos e os verbos de atividade com afetação (instrumentais). Cada uma dessas classes possui uma subclasse, sendo elas, respectivamente: a dos verbos de expressão e a dos verbos instrumentais com dois instrumentos. Evidenciamos, assim, a adequação da nossa proposta de classificação para a descrição e análise teórica do Léxico verbal do PB.

\section{REFERÊNCIAS}

ALEXIADOU, A.; ANAGNOSTOPOULOU, E. Manner vs. result complementarity in verbal alternations: a view from the clear alternation. In: Proceedings of the 42nd Annual Meeting of the North East Linguistic Society, 2013. p. 39-52.

ALMEIDA, V. Os verbos de remoção no português brasileiro. Belo Horizonte, MG: 2015. Monografia de bacharelado em Linguística. Faculdade de Letras da UFMG, Belo Horizonte, 37 p. 
AMARAL, L.; CANÇADO, M. Argument structure of activity verbs in Brazilian Portuguese. Semantics-Syntax Interface, v. 2, n. 2, [s.1.], 2015.

BECHIR, T. Os verbos recíprocos intransitivos no português brasileiro. Belo Horizonte, MG: 2016. Monografia de Bacharelado em Português (Estudos Linguísticos). Faculdade de Letras da UFMG, Belo Horizonte, 87 p.

CANÇADO, M.; AMARAL, L. Representação lexical de verbos incoativos e causativos no PB. Revista da Abralin, v. 9, n. 2, p. 123-147, 2010.

CANÇADO, M.; AMARAL, L. Introdução à semântica lexical: papéis temáticos, aspecto lexical e decomposição de predicados. Petrópolis: Vozes, 2016.

CANÇADO, M.; AMARAL, L.; MEIRELLES, L. Banco de Dados Lexicais VerboWeb: classificação sintático-semântica dos verbos do português brasileiro, UFMG, 2017. Disponível em: $<$ http://www.letras.ufmg.br/verboweb/>. Acesso: 14 set. 2017

CANÇADO, M.; GODOY, L.; AMARAL, L. Catálogo de verbos do português brasileiro: classificação verbal segundo a decomposição de predicados. Vol I. Verbos de mudança. Belo Horizonte: Editora UFMG, 2013.

CANÇADO, M.; GODOY, L.; AMARAL, L. Catálogo de verbos do português brasileiro: classificação verbal segundo a decomposição de predicados. Vol I. Verbos de mudança, 2 ed. Amazon. 2017.

CANÇADO, M.; GONÇALVES, A. Lexical Semantics: verb classes and alternations. In: WETZELS, L.; MENUZZI, S.; COSTA, J. The Handbook of Portuguese Linguistics. Willey/Blackwell, 2016.

DE CLERCK, B.; COLLEMAN, T.; WILLEMS, D. Introduction: a multifaceted approach to verb class. Linguistic, n. 51 (4), [s.1.], 2013, p. 663-680.

DIXON, R. M. W. A new approach to English grammar, on semantic principles. Oxford: Clarendon Press, 1992.

FILLMORE, C. The grammar of hitting and breaking. In: JACOBS, R.; ROSENBAUM, P. Readings in English Transformational Grammar. Waltham: Ginn, 1970, p. 120-133.

FONSECA, J. Verbos simétricos. Boletim de filologia. Lisboa, v. 2, [s.1.], 1984, p. 383-403. 
GODOY, L. Os verbos recíprocos no PB e a hipótese da determinação semânticolexical sobra a sintaxe. Alfa, v. 53, n. 1. [s.1.], 2009, p. 283-299.

GODOY, L. A semântica da dupla realização argumental dos verbos recíprocos. Revista do GEL, v. 7, n. 1, [s.1.], 2010, p. 95-115.

GRIMSHAW, J. Words and structure. Standford: CSLI/ University of Chicago Press, 2005.

HARLEY, H. How do verbs get their names? Denominal Verbs, Manner Incorporation andthe Ontology of Verb Roots in English. In: ERTESCHIK-SHIR, N.; RAPPOPORT, T. The Syntax of Aspect. Oxford: Oxford university Press, 2005, p. 42-64.

HASPELMATH, M. More on the typology of inchoative/causative verb alternations. In: COMRIE, B.; POLINSKY, M. Causatives and transitivity. Amsterdam: John Benjamins, 1993, p. 87-120.

HORROCKS, G.; STAVROU, M. Morphological Aspect and the Function and Distribution of Cognate Objects Across Languages. In: RAPPAPORT HOVAV, M.; DORON, E.; SICHEL, I. Lexical Semantics, Syntax, and Event Structure. Oxford: Oxford University Press, 2010, p. 284-308.

JACKENDOFF, R. Semantic Interpretation in Generative Grammar. Cambridge: MIT, 1972.

JACKENDOFF, R. Semantic Structures. Cambridge: MIT, 1990.

KEYSER, J.; ROEPER, T. On the middle and ergative constructions in English. Linguistic Inquiry, 15, p. 381-416, 1984.

KIPARSKY, P. Word formation and the lexicon. In: Proceedings of the MidAmerica Linguistics Conference, ed. F. Ingeman. University of Kansas, 3-29, 1982.

LAKOFF, G. Irregularity in Syntax. New York: Holt, Rinehart and Winston, 1970.

LEHRER, Adrienne. Checklist for verbs of speaking. Acta Linguistica Hungarica, vol. 38, n. 1-4. [s.1.], 1988, p. 143-161.

LEVIN, B. English Verb Classes and Alternations: A Preliminary Investigation. Chicago: University of Chicago Press, 1993.

LEVIN, B. What is the best grain-size for defining verbs? - Conference on Word Classes: Nature, Typology, Computational Representations. Second Triple International Conference, 24-26/03. Roma: Università Roma Ter, 2010. 
[disponível em http://web.standfor.edu/ bclevin/rome10grain.pdf - acessado em 06/08/2015]

LEVIN, B.; RAPPAPORT HOVAV, M. Wiping the slate clean: a lexical semantic exploration. Cognition, v. 41. [s.1.], 1991. p. 123-151.

LEVIN, B.; RAPPAPORT HOVAV, M. The Lexical Semantics of Verbs of Motion: The Perspective from Unaccusativity. In: ROCA, I. Thematic Structure: Its Role in Grammar. Berlin: Foris, 1992, p. 247-269.

LEVIN, B.; RAPPAPORT HOVAV, M. A Preliminary Analysis of Causative Verbs in English. Lingua, n. 92, [s.1.], 1994, p. 35-77.

LEVIN, B.; RAPPAPORT HOVAV, M. Unaccusativity: At the Syntax-Lexical Semantics Interface, Linguistic Inquiry Monograph 26, MIT Press, Cambridge, MA, 1995.

LEVIN, B; RAPPAPORT HOVAV, M. Argument Realization. Cambridge: Cambridge University Press, 2005.

MEIRELLES, L.; CANÇADO, M. Os verbos instrumentais no português brasileiro. Veredas, v. 19, n. 2. [s.1.], 2015, p. 292-309.

MOREIRA, C. Princípio de Ligação Sintaxe/Semântica: Construções Estativas. Belo Horizonte, MG: 2000. 93f. Dissertação de Mestrado, Faculdade de Letras da UFMG, Belo Horizonte.

OLIVEIRA, K. Alternâncias de objeto no português brasileiro: a alternância locativa. Relatório final de Iniciação Científica. Faculdade de Letras da UFMG, Belo Horizonte, 2016.

PARSONS, T. Events in the Semantics of English. Cambridge: MIT Press, 1990.

PESETSKY, D. Zero Syntax. Cambridge: MIT Press, 1995.

PINKER, S. Learnability and Cognition: The acquisition of argument structure. Cambridge: MIT Press, 1989.

SARTORI, B. Uma análise sintático-semântica das classes de verbos de expressão no português brasileiro. Belo Horizonte, MG: 2017. Monografia de Bacharelado em Português (Estudos Linguísticos). Faculdade de Letras da UFMG.

SCHER, A.P.; LEUNG, R. Os objetos cognatos e os modificadores adverbiais. Estudos Linguísticos, v. 35, [s.1.], 2006, p. 1668-1676. 
SILONI, T. The syntax of reciprocal verbs: an overview. In: KÖNIG, E.; GAST, V. (Ed.). Reciprocals and reflexives: cross-linguistics and theoretical explorations. Berlim: Mouton de Gruyter, 2007. p. 451-498.

VENDLER, Z. Linguistics in Philosophy. Ithaca: Cornell, 1967.

Márcia Cançado MCancado@ufmg.br

Luana Amaral LuanaLopes@ufmg.br

Letícia Lucinda Meirelles LeLuMeirelles@hotmail.com

Recebido em: 13 jan. 2018 Aceito em: 21 abr. 2018

Publicado em: 1 out. 2018 